The risk was highest and largely confined to the first 8 weeks of therapy, and phenytoin, phenobarbital, and carbamazepine were associated most frequently. Valproic acid, in combination with other drugs, and lamotrigine were less frequently associated. Glucocorticosteroid intake or radiotherapy did not increase the risk in this study. (Rzany B, Correia O, Kelly JP et al, for the International Study on Severe Cutaneous Adverse Reactions. Risk of Stevens-Johnson syndrome and toxic epidermal necrolysis during first weeks of antiepileptic therapy; a casecontrol study. Lancet June 26, 1999;353:2190-2194). (Respond: Dr Berthold Rzany, Hautklinik der Facultat fur klinische Medizin Mannheim der Universitat Heidelberg, Linikum Mannheim gGmbH, Theodor Kutzer Ufer, D-68135 Mannheim, Germany).

COMMENT. The risk of severe skin reactions with antiepileptic drug therapy is most frequently reported with phenytoin, phenobarbital, and carbamazepine, and is largely confined to the start of treatment. Lamotrigine is also a potential cause of Steven-Johnson syndrome and Lyell's syndrome (toxic epidermal necrolysis), while valproic acid is rarely associated. See Progress in Pediatric Neurology III, PNB Publ, 1997;pp143-146; and Vol II, 1994;pp107-109, for additional reports of AED-induced Stevens-Johnson syndrome.

\title{
EFFECT OF EPILEPSY SURGERY ON ASSOCIATED AUTISM
}

The outcome of pervasive developmental disorder (PDD) in 5 children who underwent epilepsy surgery at 3 to 8 years of age is reported from the Cleveland Clinic Foundation, Cleveland, OH. Four children had temporal lobe resections, and one a right temporoparietal-occipital resection. Three had focal cortical dysplasia, and 2 had tumors. At 14-47 month follow up (mean, 23 months), one child with persistent seizures had moderate improvements in development and behavior, 3 with seizure control were mildly improved, and 1 seizure free patient had worsened PPD associated with cognitive and emotional deterioration. Behavioral and cognitive changes were independent of seizure outcome. (Szabo CA, Wyllie E, Dolske $\mathrm{M}$ et al. Epilepsy surgery in children with pervasive developmental disorder. Pediatr Neurol May 1999;20:349-353). (Respond: Dr Wyllie, Head, Pediatric Epilepsy Program, Cleveland Clinic Foundation, 9500 Euclid Ave, Cleveland, $\mathrm{OH} 44195$ ).

COMMENT. Symptoms of pervasive developmental disorder may not show improvements, and are sometimes worsened, after epilepsy surgery for refractory seizures. Improvements in behavior and cognition can be associated with persistent epilepsy, whereas postoperative PPD deterioration may follow seizure control.

"Paradoxical normalization" (acute psychiatric symptoms with abrupt cessation of seizures and normalized EEG) has been observed in children with epilepsy treated with ACTH or antiepileptic drugs. (See Progress in Pediatric Neurology III, 1997;pp71-73). This syndrome was particularly common during trials of phenacemide (Phenurone) for "psychomotor" epilepsy in the early 1950s. Some drugs appear to have a greater propensity than others to cause personality disorders, and the association between epilepsy and psychosis is age-dependent. The evidence that surgical control of seizures can also induce a worsening of autistic symptoms suggests that seizure control per se is the mechanism and not a specific adverse effect of the antiepileptic drug. Further investigation of this phenomenon is indicated. 